\title{
Máquina de Turing Analógica para Ensino de Linguagens Formais e Autômatos
}

\author{
Leonardo Rebello Januário \\ Laboratório de Inteligência Aplicada \\ Universidade do Vale do Itajaí \\ Itajaí, SC, Brasil \\ rebello561@edu.univali.br
}

\author{
Gustavo Henrique Müller \\ Laboratório de Inteligência Aplicada \\ Universidade do Vale do Itajaí \\ Itajaí, SC, brasil \\ gustavomuller@edu.univali.br
}

\author{
Alex Luciano Roesler Rese \\ Laboratório de Inteligência Aplicada \\ Universidade do Vale do Itajaí \\ Itajaí, SC, Brasil \\ alexrese@univali.br
}

\author{
Thiago Felski Pereira \\ Laboratório de Inteligência Aplicada \\ Universidade do Vale do Itajaí \\ Itajaí, SC, Brasil \\ felski@univali.br
}

\author{
Rudimar Luís Scaranto Dazzi \\ Laboratório de Inteligência Aplicada \\ Universidade do Vale do Itajaí \\ Itajaí, SC, Brasil \\ rudimar@univali.br
}

\section{RESUMO}

The article describes the development of a practical device for teaching in the area of Computer Theory. In the study, an adaptation of the Turing Machine is presented, using hardware and software integration to interpret Formal Languages. Simulating an Automaton, sensors and motors are used to move the device head to the left and right and to read and write the input tape. The development of the mechanism is described in two parts, the first includes the hardware that consists of the construction and adaptation of the Turing Machine, the second the implementation of the software and communication part between both. The developed device, allows the interpretation of a binary alphabet $(0,1)$, where an input word is accepted, and as an output result, such device rejected or accepted the word.

\section{KEYWORDS}

Máquina de Turing, Linguagens Formais e Autômatos, Teoria da Computação

\section{INTRODUÇÃO}

Teoria da Computação compreende propriedades matemáticas fundamentais do hardware, software e de certas aplicações, buscando determinar o que pode ou não ser computado, considerando tempo e/ou memória [1], sendo composta por três áreas centrais de estudo: (i) Teoria da Complexidade; (ii) Teoria da Computabilidade; e (iii) Teoria de Autômatos.

A importância de disciplinas de Teoria da Computação é reconhecida no currículo de referências da Sociedade Brasileira de Computação [2] para os cursos de graduação em Ciências da Computação e Engenharia da Computação, estando presente em todos os eixos de formação para um graduando em Computação. Contudo [3-6], relatam as diversas dificuldades que os alunos têm em compreender os conceitos teóricos formais apresentados nas disciplinas que compreendem Teoria da Computação. Dentre os modelos apresentados em sala de aula, a Máquina de Turing é um bastante preciso e de propósito geral [1].

Disciplinas da área de Teoria da Computação possuem altos índices de reprovação. Porém, os conceitos apresentados por elas são fundamentais para a formação do currículo de graduação e pós-graduação na área da Computação [7].

No trabalho [3], o resultado obtido de sua pesquisa foi de que os acadêmicos que cursam as disciplinas de Linguagens Formais e Autômatos, muitas vezes saem sem compreender a sua importância e como aplicar seus conceitos em outras áreas.

Este trabalho tem como objetivo apresentar o desenvolvimento de uma Máquina de Turing física, para o auxílio em sala de aula. O projeto contempla a integração software e hardware, utilizando descrições de autômatos gerados pela ferramenta de simulação de linguagens formais, JFLAP [8], para simular o funcionamento de uma Máquina de Turing por meio do controle de sensores e motores, dando assim o retorno prático ao aluno. O complemento visual fornecido pela Máquina de Turing, demonstra os princípios teóricos de maneira aplicada, auxiliando a compreensão formal teórica e instigando o estudo e curiosidade perante a disciplina.

\section{PROBLEMA}

Disciplinas de Teoria da Computação possuem índices elevados de reprovação, sendo que na Universidade Federal de Pernambuco - UFPE o índice de aprovados em média é inferior a 53\% [9]. Na Figura 1, são observados os índices de reprovação para os curso de Ciências da Computação e Engenharia de Computação nos anos de 2014 à 2017. Para a formação de acadêmicos e egressos, em cursos de Computação, as disciplinas teóricas são o principal diferencial em relação aos cursos técnicos. A falta do aprendizado dos conceitos formais das disciplinas de Teoria da Computação não só prejudica o aprendizado da disciplina, como também o estudo de outras disciplinas que usam Teoria da Computação como sua base teórica.

Os materiais de estudos antigos e teóricos são de entendimento complexo e pouco atrativos para os acadêmicos [10], tais conteúdos muitas vezes são atualizados para auxiliar o ensino do acadêmico. A base de estudos é de pouco incentivo e não o mantém o fervor de ler um livro carregado de texto e com códigos complexos de exemplo. Assim, dificultando o interesse pela disciplina em questão.

Muitos materiais específicos para auxílio ao ensino do conceito da Máquina de Turing, são exclusivamente teóricos. As ferramentas de auxilio, como por exemplo JFLAP 7.1 [3], Turing Machine Simulator [11, 12], Turing Machines Implemented in JavaScript [13] e 


\begin{tabular}{|l|c|c|c|c|}
\hline \multirow{2}{*}{ Cursos } & \multicolumn{4}{|c|}{ Período } \\
\cline { 2 - 5 } & \multicolumn{2}{|c|}{2014.1 à 2015.2 } & \multicolumn{2}{c|}{2016.1 à 2017.2 } \\
\cline { 2 - 5 } & Reprovados & R. Falta & Reprovados & R. Falta \\
\hline Engenharia da Computação & $23.93 \%$ & $17.18 \%$ & $49.25 \%$ & $12.44 \%$ \\
\hline Ciências da Computação & $32.95 \%$ & $17.92 \%$ & $28.68 \%$ & $25.58 \%$ \\
\hline
\end{tabular}

Figura 1: Taxa de alunos reprovados na disciplina de Informática Teórica. [9]

Turing Machine [14] são utilizadas apenas como forma de validação de uma determinada fita de entrada ao respectivo autômato de Turing descrito. Desta forma, existe uma carência de ferramentas que trabalhem com o conceito completo da teoria de Turing, que consiste em:

(1) desenvolver a função de transferência da Máquina de Turing;

(2) determinar uma fita de entrada; e

(3) visualizar as interações que a unidade de controle realiza em relação a determinada fita de entrada.

\section{MATERIAL DESENVOLVIDO}

O projeto da Máquina de Turing Analógica contempla o desenvolvimento de uma estrutura física, sendo controlada por meio da plataforma de prototipagem Arduino [15] e um software de controle, com o objetivo de incentivar os acadêmicos de Linguagens Formais e Teoria de Autômatos a aplicar à teoria da Máquina de Turing, a aprender os conceitos apresentados em sala de aula.

\subsection{Estrutura física}

A estrutura física da Máquina de Turing foi desenvolvida para estimular e auxiliar no aprendizado da teoria apresentada em sala de aula, onde está estrutura visa demonstrar ao acadêmico:

- a leitura em uma célula da fita de entrada;

- a escrita em uma célula da fita de entrada; e

- e o movimento pelas células da fita de entrada.

A fita de entrada da Máquina de Turing desenvolvida, é representada por um conjunto de fitas de LED (3528) de tom branco frio. A fita de LED permite a construção de autômatos binários, na qual os valores variam de 1 (ligado) ou 0 (desligado). para realizar a interação com os LED's, se utilizou de um fotoresistor LDR de $5 \mathrm{~mm}$. O LDR realiza a leitura dos LED's e com base na variação de sua resistência, determina qual o valor presente no estado. O intervalo do sensor de luminosidade está entre $10 \mathrm{~K} \Omega$ à $10 \mathrm{M} \Omega$ para o LED ligado e desligado, respectivamente. Para realizar o movimento do cabeçote de leitura entre as células da fita de entrada, foi utilizado uma impressora (Epson Stylus c65). A estrutura já possui motores e sensores que possibilitam o desenvolvimento de um cabeçote móvel, que percorre entre todas as células da fita de LED. Na Figura 2, é visualizada a estrutura desenvolvida no projeto e seus principais componentes e a forma com que se integram.

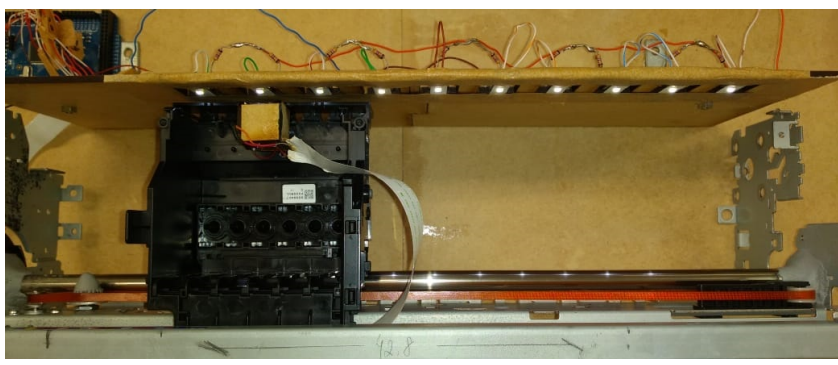

Figura 2: Estrutura Física mostrando a unidade de controle e fita de entrada. Autoral.

\subsection{Software}

O software desenvolvido no projeto, tem como objetivo integrar uma ferramenta de simulação de Linguagens Formais, o JFLAP, e disponibilizar uma interface ao usuário no qual permita a escolha do arquivo a ser utilizado na simulação e determinar a fita de entrada inicial, figura 3. O software é responsável pelas seguintes tarefas:

- realizar a análise dos autômatos gerados pelo JFLAP;

- controlar o algoritmo de controle da máquina; e

- facilitar o uso da máquina por meio de uma interface de controle interativa.

Os arquivos gerados pelo JFLAP possuem uma extensão própria (.jff), similar à de um arquivo XML. Com a análise destes arquivos, obtêm-se os estados, transições, estado inicial e estados terminais do autômato em questão. O algoritmo desenvolvido usufrui das informações coletadas pelo arquivo do JFLAP para simular uma Máquina de Turing e interagir com a estrutura física desenvolvida. Na Figura 3, é visualizada a interface desenvolvida ao longo da pesquisa, que permite a seleção do arquivo .jff e da escolha da fita de entrada inicial.

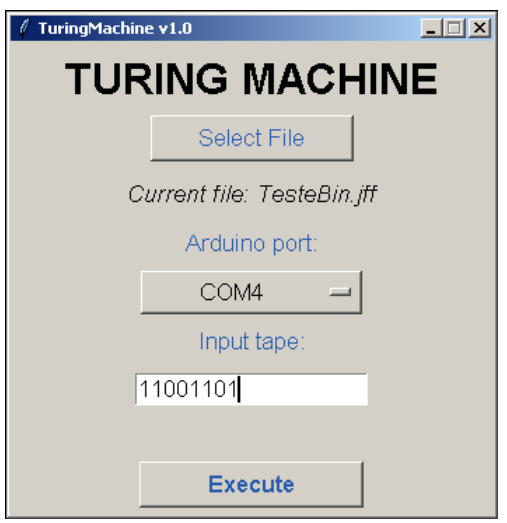

Figura 3: Interface do software desenvolvida no projeto da máquina de Turing física. Autoral.

\section{CONSIDERAÇÕES FINAIS}

Foram desenvolvidos dois materiais abordando como tema o foco na de Teoria de Autômatos. A primeira consiste na construção de uma estrutura física que representa o funcionamento da Máquina 
de Turing. A segunda o desenvolvimento de um programa capaz de realizar a interpretação e saída de ferramenta JFLAP, assim como a comunicação com a estrutura física. O objetivo em curto prazo é auxiliar os alunos da disciplina de Linguagens Formais e Autômatos no curso de Ciência da Computação e Engenharia de Computação na Universidade do Vale do Itajaí - UNIVALI. Pretende-se agora, realizar os testes e analisar os resultados em sala de aula do uso do material desenvolvido.

\section{AGRADECIMENTOS - ACKNOWLEDGMENTS}

Os autores agradecem ao Estado de Santa Catarina por ceder uma bolsa de pesquisa do artigo 170 da constituição do estado.

\section{REFERÊNCIAS}

[1] Michael Sipser. Introdução à Teoria da Computação. $2^{\text {a }}$ edition, 2005. ISBN 8522104999.

[2] Avelino F. Zorzo, Dalto Nunes, Ecivaldo S. Matos, Igor Steinmacher, Jair C. Leite, Renata Araujo, Ronaldo C. M. Correia, and Simone Martins. Referenciais de Formação para os Cursos de Graduação em Computação. 2017. ISBN 978-85-7669424-3.

[3] Eric Gramond and Susan H. Rodger. Using jflap to interact with theorems in automata theory. Trigésimo Simpósio Técnico SIGCSE sobre Educação em Ciência da Computação, 1999.
[4] Carlos Chesñevar, Maria González, and Ana Maguitman. Didactic strategies for promoting significant learning in formal languages and automata theory. ACM SIGCSE Bulletin, 36:7-11, 09 2004. doi: 10.1145/1007996.1008002.

[5] Yandre M. e G. da Costa, Rafael C. de Meneses, and Flavio R. Uber. Uma ferramenta para auxílio didático no ensino de teoria da computação. Congresso da Sociedade Brasileira de Computação, 2008.

[6] Mukta Goyal and Shelly Sachdeva. Enhancing theory of computation teaching through integration with other courses. International fournal of Recent Trends in Engineering, pages 137-140, 2009.

[7] MEC. Referenciais nacionais de cursos de graduação (licenciatura e bacharelado). http://portal.mec.gov.br/index.php?option=com content\&view=article\& id=13812\&itemid=995, 2009.

[8] Susan H. Rodger. Jflap7.1. URL http://www.jflap.org/jflaptmp/.

[9] Universidade Federal de Pernambuco. Disciplinas que mais reprovam na ufpe. https://www.ufpe.br/documents/38954/1317627/Relat\%C3\%B3rio+ Disciplinas+que+mais+reprovam+2014+a+2017.pdf/f1ba5ea6-dbdb-4edd-b5e79b993f0ee838, 2018

[10] Marcus Vinícius Midena Ramos. Ensino de linguagens formais e autômatos nos cursos superiores de computação. Revista de Computação e Tecnologia, 1:22-34, 2009.

[11] Anthony Morphett. Turing machine simulator, 2014. URL http://morphett.info/ turing/turing.html.

[12] Paul Rendell. Turing machine simulator, 2015. URL http://rendell-attic.org/gol/ TMapplet/.

[13] Andrew Hodges. Turing machines implemented in javascript, 2012. URL https: //www.turing.org.uk/book/update/tmjavar.html.

[14] Martin Ugarte. Turing machine, 2017. URL https://turingmachinesimulator.com/.

[15] Arduino. Arduino, 2019. URL https://www.arduino.cc/. 